\title{
Comparação entre Puérperas Fumantes e Ex-Fumantes com Relação ao Tempo de Amamentação e suas Consequências sobre a Saúde dos Recém-Nascidos \\ Comparison between Postpartum Smokers and Ex-Smokers as to Breastfeeding Duration and its Impact on the Health of Newborns \\ Comparación entre los Fumadores y ex Fumadores Después del Parto con Respecto a la Duración de la Lactancia Materna y su Impacto en la Salud de los Recién Nacidos
}

\author{
Adriani Oliveira Galão', Bruno Rocha de Macedo², Rafaela Vanin Pinto Ribeiro ${ }^{3}$, Roberto Vanin Pinto Ribeiro ${ }^{4}$, Carla Maria De Martini Vanin ${ }^{5}$
}

\section{Resumo}

Introduçáo: A influência nociva do tabagismo no período gestacional e na amamentação é amplamente descrita na literatura. Objetivo: Avaliar a relação entre o tempo de amamentação e o tabagismo entre as puérperas fumantes e ex-fumantes do Hospital de Clínicas de Porto Alegre e suas consequências para a saúde dos bebês. Métodos: Estudo transversal com aplicação de questionário via telefone em puérperas fumantes do HCPA, divididas em 2 grupos: mulheres que não fumaram (G1) e que fumaram (G2) após o parto. Variáveis contínuas foram descritas por medidas de tendência central e dispersão; variáveis categóricas, por frequências absolutas e relativas. As médias foram comparadas com $t$ de Student e as complicações entre os grupos com Qui-quadrado. Resultados: Avaliadas 154 puérperas: 75 (G1) e 79 (G2). A idade não diferiu entre os grupos (26,0 e 24,7 anos); 67,5\% tinham feito pré-natal e, destas, metade não fumou no pós-parto. Somente $51,3 \%$ receberam informação médica de que o fumo poderia trazer complicaçôes para ela e seu bebê. Não houve diferença estatisticamente significativa com relação a problemas ou intercorrências respiratórias nos bebês. As puérperas amamentaram em média 7,2 (G1) e 6,2 (G2) meses e não houve diferença estatisticamente significativa entre os grupos. No grupo G2 houve uma tendência à interrupção da amamentação por causas respiratórias $(\mathrm{p}=0,058)$. Conclusão: Não houve alterações significativas com relação a problemas respiratórios nos bebês ou parada da amamentação em puérperas fumantes. No entanto, o tabagismo é um preocupante problema de Saúde Pública e deve receber atenção constante nesse grupo especial de mulheres.

Palavras-chave: Tabagismo; Aleitamento Materno; Tabaco; Período Pós-Parto

Instituição de realização do estudo: Hospital de Clínicas de Porto Alegre (HCPA), Serviço de Ginecologia e Obstetrícia do Departamento de Ginecologia e Obstetrícia da Faculdade de Medicina (FAMED) da Universidade Federal do Rio Grande do Sul (UFRGS).

Patrocinador: Fundo de Incentivo à Pesquisa e Eventos-FIPE/HCPA.

${ }^{1}$ Doutora em Clínica Médica e Ciências da Saúde da PUCRS. Professora Adjunta do Departamento de Ginecologia e Obstetrícia da FAMED da UFRGS. E-mail: adrianig@brturbo.com.br. Porto Alegre (RS), Brasil.

${ }^{2}$ Acadêmico da FAMED da UFRGS. E-mail: brunomacedobrm@hotmail.com. Porto Alegre (RS), Brasil.

${ }^{3}$ Acadêmica da FAMED da UFRGS. E-mail: rafavan@terra.com.br. Porto Alegre (RS), Brasil.

${ }^{4}$ Acadêmico da FAMED da UFRGS. E-mail: rpribeiro@terra.com.br. Porto Alegre (RS), Brasil.

${ }^{5}$ Doutora em Clínica Médica pela Universidade de Toronto, Canadá; UFRGS, Brasil. Professora Adjunta do Departamento de Ginecologia da Universidade Federal de Ciências da Saúde de Porto Alegre (UFCSPA). E-mail: carlavanin@terra.com.br. Porto Alegre (RS), Brasil.

Enderę̧o para correspondência: Adriani Oliveira Galâo. Rua Inconfidência, 1.603 - Canoas (RS), Brasil. CEP: 92020-320. 


\section{INTRODUÇÃO}

A influência nociva do tabagismo no período gestacional e na amamentação é amplamente descrita na literatura médica. $\mathrm{O}$ tabagismo materno não gera somente danos ao bebê durante a sua vida intrauterina como, por exemplo, restrição do crescimento, baixo peso, ruptura prematura de membranas e descolamento prematuro de placenta. Seus efeitos também afetam o período pósnatal, incluindo o término precoce da lactação e suas consequências sobre o desenvolvimento da criança ${ }^{1-2}$.

Segundo a Organização Mundial da Saúde, há uma tendência de aumento do tabagismo entre as mulheres, e sabe-se que o seu início coincide temporalmente com o período de idade fértil ${ }^{3}$; porém, apesar de algumas mulheres abandonarem o fumo durante a gravidez, muitas delas retomam o tabagismo no pós-parto imediato ou durante o período puerperal e de forma concomitante com a amamentação ${ }^{4-5}$. Sabe-se que essas gestantes geram mais gastos em saúde do que as náo fumantes ${ }^{6}$. As taxas de amamentação nos Estados Unidos seguem abaixo do recomendado pelas suas diretrizes, sendo ainda menores entre as fumantes, nas quais existe uma percepção de menor produção de leite ${ }^{7-8}$. O fumo está associado a uma duração menor do período de amamentação: menor volume e um menor teor de gordura no leite são alguns fatores envolvidos. Além disso, o sono do lactante é afetado, o que também pode explicar a mudança no seu padrão alimentar. $\mathrm{O}$ estilo de vida saudável, o alto nível educacional e a experiência de amamentação positiva prévia são características que melhoraram significativamente as taxas de manutenção da amamentação".

Estudos norte-americanos apontam que a prevalência de fumo na gestação varia de $8,8 \%$ a $20,3 \%{ }^{10-11}$. No Brasil, existe uma prevalência entre $23 \%$ e $25 \%$, detectando os seguintes fatores de risco: a raça branca, o baixo nível socioeconômico, a baixa escolaridade e o estado civil solteira ${ }^{1,12}$. A não realização de pré-natal é mais frequente na população de fumantes, o que implica risco aumentado de infecçôes maternas, complicações para o feto e consumo de drogas ilícitas ${ }^{13}$. Máes tabagistas expõem seus filhos a um risco de desenvolvimento de Síndrome da Morte Súbita do Lactente ${ }^{14-16}$, de Diabetes Mellitus tipo 2 no adulto jovem ${ }^{17}$, de asma ${ }^{18}$ e de efeitos cognitivos deletérios ${ }^{2}$. Lactentes de usuárias de mais de cinco cigarros por dia tendem a apresentar sintomas que, por vezes, não são notados como sendo sintomas de abstinência fetal ao tabagismo e que podem ser facilitadores da interrupçâo da amamentação, como cólicas e choros ${ }^{19}$.

A nicotina diminui a produção do leite materno através da inibição da secreção de prolactina e, por isso, a redução do tabagismo é capaz de aumentar substancialmente o tempo de amamentaçáo ${ }^{3,13}$; porém há estudos que sugerem que as diferenças sociais e comportamentais entre fumantes e não fumantes podem ser mais importantes do que possíveis efeitos fisiológicos do fumo 5 . Entre os efeitos imediatos dessa substância, pode ocorrer redução importante das horas de sono do lactente ${ }^{20}$; mas, apesar disso, o tabagismo não contraindica a lactação, pois os benefícios desta são maiores do que os eventuais riscos do desmame precoce. A amamentação por mais de três meses atenua o efeito de infecçóes respiratórias de repetição e o risco de desenvolver asma entre 1 e 10 anos de idade ${ }^{21}$.

Tendo em vista os inúmeros benefícios que o período de aleitamento materno proporciona ao bebê e os malefícios amplamente comprovados que o cigarro acarreta nesta fase do desenvolvimento da criança, este estudo avaliou a relação entre o tempo de amamentação e o tabagismo entre as puérperas fumantes e ex-fumantes do Hospital de Clínicas de Porto Alegre (HCPA) e suas consequências para a saúde dos bebês.

\section{MÉTODO}

Estudo prospectivo e transversal, com aplicação de questionários via telefônica nas puérperas do HCPA, no período de outubro de 2007 a março de 2008 . O projeto obteve a aprovaçáo da Comissão de Ética e Pesquisa do HCPA, $n^{\circ} 07-511$, e todas assinaram previamente o termo de consentimento livre e esclarecido.

As pacientes incluídas eram hígidas, com gestaçóes a termo (acima de 37 semanas), que tiveram seus recém-nascidos no HCPA, sem intercorrências durante o pré-natal ou parto. Foram excluídas todas as pacientes que não puderam ser contatadas durante o período do estudo ou que tiveram seus dados de prontuários incompletos.

O protocolo incluiu dados de identificação, anamnese sobre o tabagismo no período pós-parto e suas complicaçôes sobre a saúde dos recém-nascidos, hábito de aleitamento preferencialmente materno (definido como pelo menos cinco vezes ao dia com leite materno).

As variáveis analisadas no estudo foram: (a) Dados demográficos: idade, estado civil, raça, grau de instrução e paridade; (b) Anamnese: uso do cigarro durante o período puerperal, informaçóes sobre tratamentos para o abandono do tabagismo, tempo de abstinência ou retorno ao tabagismo após o parto e quantidade de cigarros ao dia; (c) Dados perinatais: diagnósticos patológicos e internação da criança no período pós-parto; (d) Dados puerperais: tempo de amamentaçáo predominantemente com leite materno, causas da parada dessa amamentação.

Para fins estatísticos, a amostra foi dividida em dois grupos: (1) mulheres que não fumaram após o parto e (2) mulheres que fumaram após o parto. As variáveis contínuas foram descritas por medidas de tendência central e dispersão (média e desvio-padrão ou mediana e intervalo interquartil, conforme os dados que apresentem distribuição normal ou não), e os grupos 
foram comparados com os testes $t$ de Student e MannWhitney (com nível de significância $p<0,05$ ). As variáveis categóricas, como frequências absolutas e relativas, e os dados dos dois grupos foram confrontados usando o teste Exato de Fisher e Qui-quadrado.

\section{RESULTADOS}

A população estudada é decorrente de um estudo anterior que avaliou o tabagismo na gestação em uma amostra de 346 puérperas $^{22}$. Dessa amostra inicial, entrevistou-se, via telefone, um total de 154 puérperas, com uma perda de seguimento de 55\%. Das pacientes entrevistadas, $49 \%(\mathrm{n}=75)$ não fumaram no período pós-parto (grupo 1) e 51\% ( $\mathrm{n}=79)$ fumaram no período puerperal (grupo 2).

As características demográficas são apresentadas na Tabela 1. A média de idade foi 25 anos, com intervalo entre 16-45 anos, e não diferiu entre os grupos. Do grupo estudado, $61 \%$ das pacientes eram brancas, $58 \%$ tinham segundo e/ou terceiro grau de instrução e 67,5\% realizaram pré-natal. Das que fizeram acompanhamento durante a gestaçáo, ou seja, com pelo menos seis consultas, metade não fumou no pós-parto.

Somente $51,3 \%$ das pacientes receberam a informação médica na gestação de que o fumo poderia trazer complicaçóes para seu filho na amamentação. Após o parto e o período da amamentaçáo, somente três mulheres afirmaram ter feito algum tratamento para abandonar o fumo. Com relação ao estado civil, entre as solteiras, $40 \%$ delas nunca pararam de fumar no período de amamentação; entre as casadas, $60 \%$ não fumaram no mesmo período $(\mathrm{p}<0,001)$.
Com relaçáo aos dados de acompanhamento perinatal (Tabela 2), não houve diferença estatisticamente significativa com relação a problemas apresentados no nascimento ou internaçóes. Após o nascimento, 46 crianças $(30 \%)$ tiveram diagnóstico de alguma doença; destas, $34(22,1 \%)$ apresentaram algum problema respiratório (bronquiolite, asma, bronquite) e 27 (17,5\%) internaram por esta causa, não havendo diferenças entre os grupos. As pacientes amamentaram em média 6,7 meses e não houve diferença estatisticamente significativa quanto ao tempo da amamentação nos grupos. Classificou-se o motivo de interrupçáo da amamentação como sendo relacionado ao fumo (diminuição do leite, doenças respiratórias da criança, necessidade de complementação do leite materno e não ganho adequado de peso do lactente) e outras causas (desejo da máe ou criança, volta ao trabalho, uso de medicaçóes maternas que contraindiquem a amamentaçáo ou patologias mamárias)

Do total da amostra, 108 crianças (70\%) foram expostas diariamente ao fumo domiciliar (algum familiar próximo como pai ou avós); apesar disso, não houve diferença estatisticamente significativa no aparecimento de doenças ou necessidade de internaçóes no período após o nascimento.

\section{DISCUSSÃO}

A literatura afirma que a prevalência de gestantes e puérperas fumantes são de $23 \%$ a $25 \%$ e que, dentre essas, a maioria é branca, de baixo nível socioeconômico, baixa escolaridade e solteira ${ }^{3,12-13}$. Os dados demográficos deste trabalho diferiram da literatura, pois a cor da pele, o grau de instrução e a presença de pré-natal não apresentaram

Tabela 1. Características dos grupos estudados

\begin{tabular}{|l|c|c|c}
\hline \multicolumn{1}{c|}{ Variável } & $\begin{array}{c}\text { Grupo 1: } \\
\text { não fumaram após o parto } \\
(\mathbf{n = 7 5 )}\end{array}$ & $\begin{array}{c}\text { Grupo 2: } \\
\text { fumaram após o parto } \\
(\mathbf{n = 7 9 )}\end{array}$ & p \\
\hline $\begin{array}{l}\text { Idade (anos) } \\
\text { Cor da pele (n\%) } \\
\text { Branca }\end{array}$ & $26,0 \pm 6,3$ & $24,7 \pm 6,3$ & $0,709^{\mathrm{a}}$ \\
$\quad$ Não branca & $49(52 \%)$ & $45(48 \%)$ & \\
$\begin{array}{l}\text { Instrução (n\%) } \\
\text { Analfabeta e instrução } \\
\text { primária }\end{array}$ & $26(43 \%)$ & $34(57 \%)$ & $0,287^{\mathrm{b}}$ \\
Instrução secundária/ \\
terciária
\end{tabular}

${ }^{a}=$ teste $t^{b}=$ teste Qui-quadrado. 
Tabela 2. Características dos recém-nascidos durante a amamentação nos grupos

\begin{tabular}{|c|c|c|c|}
\hline Variável & $\begin{array}{c}\text { Grupo 1: } \\
\text { não fumaram após o parto } \\
(n=75)\end{array}$ & $\begin{array}{l}\text { Grupo 2: } \\
\text { fumaram após o parto } \\
(n=79)\end{array}$ & $\boldsymbol{p}$ \\
\hline \multicolumn{4}{|l|}{ Problemas: } \\
\hline Nenhum & 57 (76\%) & $51(64,5 \%)$ & \\
\hline Respiratórios & $13(17,3 \%)$ & $21(26,5 \%)$ & $0,294^{a}$ \\
\hline Outros & $5(6,7 \%)$ & $7(9,0 \%)$ & \\
\hline \multicolumn{4}{|l|}{ Internações: } \\
\hline Nenhum & $51(68 \%)$ & $59(74,6 \%)$ & $0,647^{a}$ \\
\hline Respiratórios & $15(20 \%)$ & $12(15,2 \%)$ & \\
\hline Outros & 9 (12\%) & $8(10,2 \%)$ & \\
\hline $\begin{array}{l}\text { Tempo de amamentação } \\
\text { (meses) }\end{array}$ & $7,2 \pm 4,7$ & $6,2 \pm 4,3$ & $0,472^{b}$ \\
\hline \multicolumn{4}{|l|}{$\begin{array}{l}\text { Motivo de interrupção da } \\
\text { amamentação }\end{array}$} \\
\hline Relacionado ao fumo & $11(14,7 \%)$ & $23(29,1 \%)$ & $0,058^{a}$ \\
\hline Outras causas & $35(46,6 \%)$ & $33(41,8 \%)$ & \\
\hline
\end{tabular}

${ }^{\mathrm{a}}=$ teste $\mathrm{t}^{\mathrm{b}}=$ teste Qui-quadrado.

significância estatística entre os grupos no pós-parto com relaçáo à manutenção do tabagismo durante o período de amamentação. Esses dados demográficos mostraram-se relevantes para o conhecimento do perfil das puérperas fumantes do HCPA para posterior planejamento de estratégias de tratamento para essas pacientes.

Os dados deste estudo foram obtidos através de entrevista telefônica e preenchimento de questionários. Com isso, houve uma elevada perda de seguimento, porém esperada. Relacionou-se isto ao fato de nossas pacientes trocarem frequentemente de endereço ou telefone num curto espaço de tempo entre os estudos ${ }^{22}$. Esse tipo de abordagem, por vezes, traz maior fragilidade aos dados que poderiam ser subestimados com taxas elevadas de falso-negativos ou incorrer em viés recordatório, como em estudo semelhante ${ }^{23}$. Dosagens de metabólitos da nicotina, como a cotinina, poderiam ser dosadas nas puérperas ou nos recém-nascidos para a confirmação das informaçôes do uso de fumo no período do puerpério ${ }^{24}$. Pesquisas mostram que lactantes amamentados por mães fumantes possuem níveis urinários de cotinina dez vezes maiores do que aqueles sem aleitamento materno, mas com exposição passiva ao fumo; e ainda 50 vezes maiores do que os não expostos ao fumo, independente da alimentação ${ }^{2}$.

A literatura aponta que a maioria das mulheres que fazem o pré-natal chega à primeira consulta sem histórico de tabagismo ou já tendo abandonado o cigarro antes ou imediatamente ao descobrirem-se grávidas ${ }^{9}$. Com relação à realização de pré-natal como fator protetor para a manutenção do fumo no período da amamentação, este estudo não apontou diferença no sentido de proteção. Parece que talvez nosso pré-natal não seja eficiente no que se refere a informar nossas puérperas adequadamente sobre os riscos de fumar e sua relação com a amamentação, pois somente $51 \%$ das pacientes receberam alguma informação sobre isso em suas visitas de pré-natal. Essas orientaçôes devem ser encorajadas por todo e qualquer agente de saúde.

A literatura demonstra maior risco de problemas respiratórios e internaçôes entre recém-nascidos de mães que fumam durante a amamentaçáo ${ }^{13,21}$. Além do efeito direto do fumo, parece haver uma modificação na colonização da via aérea inferior por patógenos, aumentando a frequência e intensidade das doenças de etiologia infecciosa na faixa pediátrica ${ }^{25}$; no entanto, entre as pacientes entrevistadas, isso não foi significativamente diferente entre os grupos.

Entre as puérperas fumantes, somente três disseram ter feito algum tratamento durante a gravidez para parar de fumar, duas o fizeram com medicação homeopática e outra com cloridrato de bupropiona, que tem risco $\mathrm{B}$ na gestação e amamentação. Isto comprova como são tratadas inadequadamente as puérperas fumantes $\mathrm{e}$ deixam de oferecer inúmeras opçôes de tratamentos que vêm sendo discutidas na literatura mundial ${ }^{2}$. $\mathrm{O}$ incentivo ao abandono do fumo é o mínimo (até 3 minutos de informação sobre riscos por exemplo); evidências sugerem que a intervenção psicossocial deve ultrapassá-lo $(5$ a 15 
minutos de conversa com diferentes profissionais da saúde já oferece vantagem estatística). Ainda que controversa, a redução do número de cigarros consumidos por dia deve ser incentivada. Não há estudos em gestantes ou lactantes demonstrando benefício da farmacoterapia, mas recomenda-se globalmente seu uso em qualquer paciente sem contraindicação específica ao tratamento. A reposição com nicotina, por exemplo, deve ser usada sob forte supervisão e após orientação sobre os riscos do tratamento. A bupropiona, apesar de aumentar o risco de anomalias congênitas quando usada no $1^{\circ}$ trimestre, pode ser utilizada e comprovadamente aumenta as taxas de abandono do tabagismo².

Embora o tempo de amamentação seja apontado na literatura como menor nas puérperas que fumam ${ }^{3,13}$, este estudo não apontou essa diferença. Talvez isto se deva a uma cultura local que enfatiza cada vez mais o hábito de amamentar como fator de proteção aos recém-nascidos ${ }^{21}$, diferentemente do que ocorre nos Estados Unidos, onde esse hábito vem diminuindo ${ }^{7-8}$. Os motivos que levaram à interrupção da amamentação em relação ao fumo não diferiram entre os grupos e, talvez, isto se explique pelo tempo médio de amamentação ser de 6,7 meses entre os grupos e pelo fato de que é cultural que mesmo nas puérperas fumantes os benefícios da amamentação superem os riscos.

\section{CONCLUSÃO}

Convém salientar que, apesar de este estudo não ter apontado esses riscos, talvez por um viés de amostragem, o tabagismo concomitante à amamentaçáo continua sendo um problema importante de Saúde Pública com consequências que comprometem não só o Sistema de Saúde, mas também a economia da sociedade como um todo. Sendo assim, é necessário continuar, nas consultas de pré-natal, a informar sobre os riscos do tabagismo no puerpério.

Nesse sentido, campanhas de orientação devem continuar abordando esse tema, a fim de conscientizar principalmente as mães fumantes dos perigos e consequências nocivas da manutenção do tabagismo, durante a amamentação, sobre seus filhos recémnascidos.

\section{AGRADECIMENTOS}

A todos os alunos que participaram da confecção de projetos e coleta dos dados: Alícia Dorneles Dornelles, André Dias Américo, Bárbara Sabrine Kilp, Gustavo Julio Dreher, João Augusto P. Bergamaschi, Lílian Leão Arais da Silva, Maicon Antônio Carraro, Manuela Martins Costa, Rodolfo Souza da Silva, Julise A. Balvedi, Matheus Dornelles Fick e Lucas Klassmann.

\section{CONTRIBUIÇÕES}

Adriani Oliveira Galão trabalhou na concepção e desenho da pesquisa, metodologia, análise e interpretação dos dados e redação final e na Coordenação e supervisão geral do trabalho; Bruno Rocha de Macedo, Rafaela Vanin Pinto Ribeiro, Roberto Vanin Pinto Ribeiro, Carla Maria De Martini Vanin trabalharam na interpretação dos dados, análise dos dados e redaçáo final.

\section{Declaração de Conflito de Interesses: Nada a Declarar.}

\section{REFERÊNCIAS}

1. Mello PRB, Pinto GR, Botelho C. Influência do tabagismo na fertilidade, gestação e lactação. J Pediatr (Rio J.). 2001;77(4):257-64.

2. Rodriguez-Thompson D. Smoking and pregnancy [Internet] [updated 2011 Jan 17; cited 2011 Jun 24]. Available from: http://www.uptodate.com/ contents/smoking-and-pregnancy? source =search_ result\&selectedTitle $=1 \% 7 \mathrm{E} 150$

3. Instituto Nacional de Câncer (Brasil). Coordenação de Prevenção e Vigilância. Prevalência de tabagismo no Brasil: dados dos inquéritos epidemiológicos em capitais brasileiras [Internet]. Rio de Janeiro: INCA; 2004 [citado 2011 jun 24]. Disponível em: http:// bvsms.saude.gov.br/bvs/publicacoes/tabaco_inquerito_ nacional_070504.pdf

4. Stroud LR, Paster RL, Goodwin MS, Shenassa E, Buka $S$, Niaura R, et al. Maternal smoking during pregnancy and neonatal behavior: a large-scale community study. Pediatrics. 2009 May;123(5):e842-8.

5. Amir LH, Donath SM. Does maternal smoking have a negative physiological effect on breastfeeding? The epidemiological evidence. Birth. 2002 Jun;29(2):112-23.

6. Bobo JK. Tobacco use, problem drinking and alcoholism. Clin Obstet Gynecol. 2002 Dec;45(4):1169-80.

7. Goldade K, Nichter M, Nichter M, Adrian S, Tesler L, Muramoto M. Breastfeeding and smoking among lowincome women: results of a longitudinal qualitative study. Birth. 2008;35(3):230-40.

8. Liu J, Rosenberg KD, Sandoval AP. Breastfeeding duration and perinatal cigarette smoking in a population-based cohort. Am J Public Health. 2006 Feb;96(2):309-14.

9. Law KL, Stroud LR, LaGasse LL, Niaura R, Liu J, Lester BM. Smoking during pregnancy and newborn neurobehavior. Pediatrics. 2003 Jun;111(6 Pt 1):1318-23.

10. Ebrahim SH, Gfroerer J. Pregnancy-related substance use in the United States during 1996-1998. Obstet Gynecol. 2003;101(2):374-9.

11. Vega WA, Kolody B, Hwang J, Noble A. Prevalence and magnitude of perinatal substance exposures in California. N Engl J Med. 1993;329(12):850-4. 
12. Barros FC, Victora CG, Barros AJ, Santos IS, Albernaz E, Matijasevich A, et al. The challenge of reducing neonatal mortality in middle-income countries: findings from three brazilian birth cohorts in 1982, 1993, and 2004. Lancet. 2005;365(9462):847-54.

13. Bada HS, Das A, Bauer CR, Shankaran S, Lester BM, Gard CC, et al. Low birth weight and preterm births: etiologic fraction attributable to prenatal drug exposure. J Perinatol. 2005 Oct;25(10):631-7.

14. Alm B, Milerad J, Wennergren G, Skjaerven R, Oyen N, Norvenius G, et al. A case-control study of smoking and sudden infant death syndrome in the Scandinavian countries, 1992 to 1995 . The Nordic Epidemiological SIDS Study. Arch Dis Child. 1998 Apr;78(4):329-34.

15. Mitchell EA, Tuohy PG, Brunt JM, Thompson JM, Clements MS, Stewart AW, et al. Risk factors for sudden infant death syndrome following the prevention campaign in New Zealand: a prospective study. Pediatrics. 1997;100(5):835-40.

16. Hoffman HJ, Damus K, Hillman L, Krongrad E. Risk factors for SIDS. Results of the National Institute of Health and Human Development SIDS Cooperative Epidemiological Study. Ann N Y Acad Sci. 1988;533:1330.

17. Montgomery SM, Ekbom A. Smoking during pregnancy and diabetes mellitus in a British longitudinal birth cohort. BMJ. 2002;324(7328):26-7.

18. Skorge TD, Eagan TM, Eide GE, Gulsvik A, Bakke PS. The adult incidence of asthma and respiratory symptoms by passive smoking in utero or in childhood. Am J Respir Crit Care Med. 2005;172(1):61-6.

19. Matheson I, Rivrud GN. The effect of smoking on lactation and infantile colic. JAMA. 1989 Jan;261(1):42-3.

20. Mennella JA, Yourshaw LM, Morgan LK. Breastfeeding and smoking: short-term effects on infant feeding and sleep. Pediatrics. 2007;120:497-502.

21. Karmaus W, Dobai AL, Ogbuanu I, Arshard SH, Matthews S, Ewart S. Long-term effects of breastfeeding, maternal smoking during pregnancy, and recurrent lower respiratory tract infections on asthma in children. J Asthma. 2008;45(8):688-95.

22. Galão AO, Soder SA, Gerhardt M, Faertes TH, Krüger MS, Pereira DF, et al. Efeitos do fumo materno durante a gestação e complicaçôes perinatais. Rev HCPA. 2009;29(3):218-24.

23. WeiserTM, Lin M, Garikapaty V, Feyerharm RW, Bensyl DM, Zhu BP. Association of maternal smoking status with breastfeeding practices: Missouri, 2005. Pediatrics. 2009;124(6):1603-10.

24. McDonald SD, Perkins SL, Jodouin CA, Walker MC. Folate levels in pregnant women who smoke: an important gene/environment interaction. Am J Obstet Gynecol. 2002;187(3):620-5.

25. Samet JM, Sockrider M. Secondhand smoke exposure: effects in children [Internet]. [updated 2011 Fev 18; cited 2011 Jun 27]. Available from: http://www.uptodate. $\mathrm{com} /$ contents/secondhand-smoke-exposure-effects-inchildren?source $=$ search_result $\&$ selectedTitle $=1 \% 7 \mathrm{E} 150$ 


\begin{abstract}
Introduction: The harmful influence of tobacco smoking during pregnancy and breastfeeding is widely described in the literature. Objective: To evaluate the relationship between the duration of breastfeeding and tobacco smoking among mothers smokers and ex-smokers in the Hospital de Clinicas de Porto Alegre and its effects on their babies. Method: A cross-sectional study applying a questionnaire by telephone among mothers smokers in the HCPA, separated in two groups: women who had not smoked after delivery (G1) and those who smoked after delivery (G2). Continuous variables were described by measures of central tendency and dispersion; categorical variables by absolute and relative frequencies. Means were compared with Student- $t$ and complications between the groups with chi-square. Results: 154 mothers were evaluated, respectively, 75 in G1 and 79 in G2. Age did not differ between groups (26.0 and 24.7 years); $67.5 \%$ had prenatal care and, of these, half did not smoke during the postpartum period. Only $51.3 \%$ received medical information that smoking could cause complications for her and her baby. There was no statistically significant difference with respect to problems or respiratory problems in babies. The groups breastfed, on average, 7.2 (G1) and 6.2 (G2) months and there was no statistically significant difference between them. In group G2, there was a tendency for breastfeeding cessation due to respiratory causes $(\mathrm{p}=0.058)$. Conclusion: There were no significant changes with respect to respiratory problems in infants or early breastfeeding cessation in mothers who smoke. However, tobacco smoking is a worrisome public health problem and should receive continued attention in this special group of women. Key words: Smoking; Breast Feeding; Tobacco; Postpartum Period
\end{abstract}

\title{
Resumen
}

Introducción: La influencia perjudicial del tabaquismo durante el período de embarazo y de lactancia está ampliamente descripta en la literatura. Objetivo: Evaluar la relación entre la duración de la lactancia materna y el acto de fumar entre los fumadores y ex fumadores madres del Hospital de Clínicas de Porto Alegre y sus efectos sobre sus bebés. Método: Estudio transversal con un cuestionario por teléfono entre las mujeres fumadoras del HCPA. Se dividieron: las mujeres que no habían fumado después del parto (G1) y las que habían fumado después del parto (G2). Las variables continuas se describen con medidas de tendencia central y dispersión; las variables categóricas mediante frecuencias absolutas y relativas. Las medias se compararon con la t de Student y las complicaciones entre los grupos con Chicuadrado. Resultados: 154 mujeres evaluadas: 75 (G1) y 79 (G2). La edad no fue diferente entre los grupos (26,0 y 24,7 años), el 67,5\% tenían control prenatal y de éstos, la mitad no fumó durante el período posparto. Sólo el 51,3\% recibió la información médica que el fumar puede causar complicaciones para ella y su bebé. No hubo diferencias estadísticamente significativas con respecto a los problemas o complicaciones respiratorias en los bebés. Los grupos de lactancia materna en meses promedio de 7,2 (G1) y 6,2 (G2), y no hubo diferencias estadísticamente significativas entre ellos. En cuanto al motivo de finalización de la lactancia materna, en el grupo 2 hubo una tendencia a romperse por causas respiratorias $(\mathrm{p}=0,058)$. Conclusión: No hubo cambios significativos con respecto a los problemas respiratorios en los bebés o la interrupción temprana de la lactancia materna entre las mujeres fumadoras. Sin embargo, el tabaquismo es un problema preocupante de salud pública y debe recibir atención continua en este grupo especial de las mujeres. Palabras clave: Tabaquismo; Lactancia Materna; Tabaco; Periodo de Posparto 